\title{
ON THE RELATION BETWEEN LYAPUNOV EXPONENTS AND EXPONENTIAL DECAY OF CORRELATIONS
}

\author{
JULIA SLIPANTSCHUK, OSCAR F. BANDTLOW AND WOLFRAM JUST
}

\begin{abstract}
Chaotic dynamics with sensitive dependence on initial conditions may result in exponential decay of correlation functions. We show that for one-dimensional interval maps the corresponding quantities, that is, Lyapunov exponents and exponential decay rates, are related. For piecewise linear expanding Markov maps observed via piecewise analytic functions we provide explicit bounds of the decay rate in terms of the Lyapunov exponent. In addition, we comment on similar relations for general piecewise smooth expanding maps.
\end{abstract}

\section{INTRODUCTION}

Chaotic motion accounts on the one hand for the well-known phenomenon of sensitive dependence on initial conditions, that is, exponentially fast divergence of nearby orbits, and on the other hand for the phenomenon of decay of correlations or mixing. Both properties are intimately related with the observation that even low-dimensional chaotic systems share common features with random processes.

This intuitive picture has been used as a basis to address some of the fundamental questions arising in nonequilibrium statistical mechanics [22, 23, 15]. In fact, it is a simple exercise to show that topological mixing implies sensitive dependence on initial conditions. At the measure-theoretical level, however, relating Lyapunov exponents, the quantitative measures for sensitive dependence on initial conditions, to decay rates of correlation functions is a more involved task. For instance, it is easy to construct simple maps with finite Lyapunov exponent and arbitrarily small correlation decay (see, for example, a Markov model described in [17]).

Thus, at a quantitative level it is tempting to explore in some detail in which way the rate of correlation decay is linked with Lyapunov exponents, as both quantities are supposed to have a common origin. In more general terms and from a wider perspective this topic can be viewed as belonging to the realm of fluctuation dissipation relations for nonequilibrium dynamics, where one cause, the underlying detailed dynamical structure, is responsible for the approach to the stationary state, that is, for the decay of correlations, but, at the same time, is responsible for fluctuation properties at a microscopic level, or in our context for the sensitive dependence of initial conditions and a positive Lyapunov exponent. Furthermore, any relation between decay of correlations and Lyapunov exponents is of great practical interest, as the measurement of Lyapunov exponents, unlike the rate of correlation decay, is notoriously difficult to determine in real world experiments $[1,18]$. In [31] it was even suggested to take correlation decay rates as a meaningful approximation for Lyapunov exponents.

The problem we want to address can be illustrated by a very basic textbook example, probably considered for the first time more than two decades ago [2]. 
Consider a linear full branch map (see Figure 1) on the unit interval $I=[0,1]$, that is, a map $f: I \rightarrow I$ having a finite partition of $I$ into closed intervals $I_{k}$ with pairwise disjoint interior such that (i) for each $k$, we have $f\left(I_{k}\right)=I$, and (ii) $f$ has constant slope $\gamma_{k}$ on each $I_{k}$. The physical invariant measure is given by the Lebesgue measure and the Lyapunov exponent with respect to this measure can be expressed in terms of the slopes

$$
\Lambda=\sum_{k}\left|I_{k}\right| \ln \left|\gamma_{k}\right|
$$

with $\left|I_{k}\right|=1 /\left|\gamma_{k}\right|$ denoting the size of the interval $I_{k}$.

The exponential rate of decay for correlation functions is determined by the negative logarithm of the second largest eigenvalue in modulus of the associated Perron-Frobenius operator. In this setting it is well known (see, for example, [27]) that eigenfunctions of the Perron-Frobenius operator are given by polynomials and that the corresponding eigenvalues $\nu_{m}$ can be expressed as

$$
\nu_{m}=\sum_{k} \frac{1}{\left|\gamma_{k}\right|} \frac{1}{\gamma_{k}^{m}}=\sum_{k}\left|I_{k}\right| \frac{1}{\gamma_{k}^{m}} \quad(m \geq 0),
$$

with largest eigenvalue $\lambda_{0}=\nu_{0}=1$ and the subleading eigenvalue $\lambda_{1}$ being the second largest in modulus, $\left|\lambda_{1}\right|=\max \left\{\left|\nu_{1}\right|, \nu_{2}\right\}$. Thus, correlation functions of sufficiently smooth observables decay typically at an exponential rate $\alpha=-\ln \left|\lambda_{1}\right|$. Since $\left|\lambda_{1}\right| \geq \nu_{2}>0$ we obtain an upper bound for the decay rate $\alpha \leq-\ln \nu_{2}$ which can now be related to the Lyapunov exponent (1). If we apply Jensen's inequality to the convex function $\varphi(x)=-\ln (x)$ we end up with

$$
\alpha \leq-\ln \sum_{k}\left|I_{k}\right| \frac{1}{\gamma_{k}^{2}} \leq \sum_{k}\left|I_{k}\right|\left(-\ln \frac{1}{\gamma_{k}^{2}}\right)=2 \Lambda .
$$

The estimate of the decay rate in (2) has been based on $\nu_{2}$ which contains positive terms only, even if the slopes have different signs. As a result the upper bound is given by twice the Lyapunov exponent. If all slopes have the same sign, say $\gamma_{k}>1$, then $\nu_{1}$ determines the subleading eigenvalue and the Lyapunov exponent itself yields an upper bound for the decay rate, that is, $\alpha \leq \Lambda$.

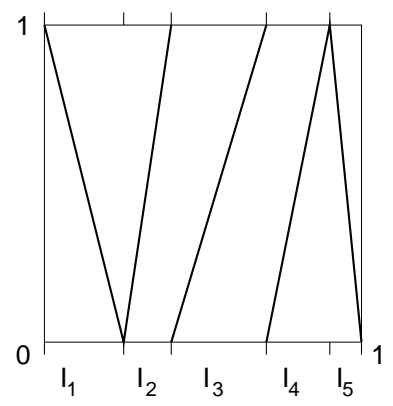

FiguRE 1. Diagrammatic view of a linear full branch map.

In this article we will address the question to which extent this simple reasoning can be generalised to a larger class of systems. From a mathematical perspective there is a considerable amount of literature on the existence of invariant measures of one-dimensional maps, the corresponding Lyapunov exponent, estimates for the 
spectra of the associated Perron-Frobenius operator, and a possible relation between Lyapunov exponents and the decay of correlation functions [12]. Expansiveness of the underlying map $f: I \rightarrow I$ is to some extent the key ingredient to establish a physical measure $\mu$, that is, an invariant measure which asymptotically characterises a large set of orbits, or in formal terms an invariant ergodic probability measure which is absolutely continuous with respect to Lebesque measure (see, for example, $[25,21,3,10])$.

The Lyapunov exponent for a Lebesque typical point $x \in I$ is given by

$$
\Lambda=\int_{I} \ln \left|f^{\prime}(x)\right| d \mu=\lim _{n \rightarrow \infty} \frac{1}{n} \ln \left|\left(f^{n}\right)^{\prime}(x)\right| .
$$

Regarding decay rates for correlation functions, a substantial part of the problem consists in giving the notion of the rate of correlation decay a proper meaning (see, for example, [12]). With the standard definition of a correlation function for two observables $\varphi$ and $\psi$,

$$
C_{\varphi, \psi}(n)=\int_{I} \varphi\left(f^{n}(x)\right) \psi(x) d \mu-\int_{I} \varphi(x) d \mu \int_{I} \psi(x) d \mu,
$$

the exponential decay rate $\alpha_{\varphi, \psi}$ governing the asymptotic behaviour of the correlation function may be formally introduced by

$$
\alpha_{\varphi, \psi}=\sup \left\{s: \limsup _{n \rightarrow \infty}\left|\exp (s n) C_{\varphi, \psi}(n)\right|<\infty\right\} .
$$

The rate $\alpha_{\varphi, \psi}$ is sensitive to the choice of $\varphi$ and $\psi$ and can be made arbitrarily small or large by special choices for the observables [13]. However, it is possible to define a rate of decay with respect to "typical" observables from some linear space $\mathcal{H}$

$$
\alpha_{\mathcal{H}}=\inf \left\{\alpha_{\varphi, \psi}: \varphi, \psi \in \mathcal{H}\right\} .
$$

We will call this quantity the mixing rate. Note that this rate still depends, for instance, on the degree of smoothness shared by the observables in $\mathcal{H}$.

One approach for determining bounds on the mixing rate $\alpha_{\mathcal{H}}$ relies on reformulating (4) in terms of the Perron-Frobenius operator. The rate $\alpha_{\mathcal{H}}$ is then determined by the subleading eigenvalue or by the essential spectral radius of the Perron-Frobenius operator. The desired relation between the Lyapunov exponent and the mixing rate expressed in (2) then becomes a lower bound for the subleading eigenvalue. There is a considerable body of literature on upper bounds for spectral values (see, for example, $[25,21,10]$ ) providing useful tools to establish ergodic properties of dynamical systems. However, to the best of our knowledge hardly any nontrivial lower bounds exist (see, however, [28], where an exponential lower bound for correlation functions of suspension semiflows is given).

The main tool to establish the desired inequality consists in studying the properties of the generalised Perron-Frobenius operator $\mathcal{L}_{\beta}$ with potential $-\beta \ln \left|f^{\prime}\right|$,

$$
\left(\mathcal{L}_{\beta} h\right)(x)=\sum_{y \in f^{-1}(x)} \frac{h(y)}{\left|f^{\prime}(y)\right|^{\beta}} .
$$

For $\beta=1$ this expression reduces to the Perron-Frobenius operator which has leading eigenvalue one. The subleading eigenvalue $\lambda_{1}$ (or the essential spectral radius, if no subleading eigenvalue exists) determines the mixing rate $\alpha_{\mathcal{H}}=-\ln \left|\lambda_{1}\right|$. In addition, the derivative of the largest eigenvalue $\nu_{0}(\beta)$ of $(5)$ with respect to $\beta$, 
that is, the derivative of the topological pressure, determines the Lyapunov exponent (see, for example, [8] for a basic exposition). Finally, the required estimate follows from the convexity of the topological pressure and gives a lower bound for the subleading eigenvalue of the Perron-Frobenius operator.

The main challenge is, of course, to put these ideas into practice. Restricting to piecewise linear Markov maps considerably reduces the need to worry about mathematical subtleties, as the operator $\mathcal{L}_{\beta}$ admits finite-dimensional matrix representations when considering observables consisting of piecewise analytic functions. Thus, at a computational level all technical details reduce to straightforward matrix manipulations (see Section 2), allowing us to keep the presentation elementary and, at the same time, making the underlying ideas transparent. To keep the presentation self-contained basic properties of piecewise linear Markov maps together with properties of $\mathcal{L}_{\beta}$ are summarised in the Appendix. In Section 3 we are addressing the question whether the bounds presented in Section 2 hold for general (non-linear) expanding Markov maps. We have compelling evidence that the estimate breaks down if analytic observables are considered (see also [11]), but the validity of our proposition can be restored if observables of bounded variation are considered.

\section{Piecewise linear Markov maps}

Let us consider a topologically mixing piecewise linear expanding Markov map $f: I \rightarrow I$ with respect to the partition $\mathcal{P}=\left\{I_{1}, \ldots I_{N}\right\}$ of the interval $I$ (see Definition A.1 for a formal account). Denote by $f_{k}:=f_{I_{k}}$ the $k$-th branch of $f$. A nice property of such maps is that the corresponding transfer operators have finite matrix representations. In fact, the space of piecewise polynomial functions of degree less than $M$, that is, the space of functions of the form $x \mapsto$ $\sum_{k=1}^{N} \sum_{m=0}^{M} a_{k m} x^{m} \chi_{k}(x)$, where $\chi_{k}$ is the characteristic function of the interval $I_{k}$, is an $\mathcal{L}_{\beta}$-invariant subspace. While it appears to be difficult to trace the earliest reference for this result, to the best of our knowledge the first experimentally relevant application appeared in the context of power spectra for intermittent dynamics (see $[27,30])$.

Now, in the natural basis of piecewise monomials, the operator $\mathcal{L}_{\beta}$ restricted to the invariant subspace mentioned above is represented by the $(M+1)^{N} \times(M+1)^{N}$ block upper triangular matrix

$$
\left(\begin{array}{cccc}
T^{(00)}(\beta) & T^{(01)}(\beta) & \cdots & T^{(0 M)}(\beta) \\
0 & T^{(11)}(\beta) & \ddots & \vdots \\
\vdots & \ddots & \ddots & T^{(M-1 M)}(\beta) \\
0 & \cdots & 0 & T^{(M M)}(\beta)
\end{array}\right) .
$$

A calculation similar to the one used to obtain the matrix representation $T^{(00)}(1)$ of $\mathcal{L}_{1}$ on the space of piecewise constant functions (see, for example, [9, p. 176]), shows that the matrix elements of the block matrices $T^{(m n)}(\beta)$ are given in terms of the slopes $\gamma_{k}$, the intercepts $d_{k}$ of the branches $f_{k}$ of the map $f$, and the topological transition matrix $\left(A_{k l}\right)_{1 \leq k, l \leq N}$ induced by $f$ and $\mathcal{P}$ (see (16)) as follows:

$$
T_{k l}^{(m n)}(\beta)=\frac{A_{l k}}{\left|\gamma_{l}\right|^{\beta} \gamma_{l}^{n}} \cdot\left(-d_{l}\right)^{n-m}\left(\begin{array}{c}
n \\
n-m
\end{array}\right) .
$$


The eigenvalues are determined by the diagonal blocks $T^{(m m)}(\beta)$ with matrix elements given by the first factor in (7).

Note that since the underlying map is assumed to be topologically mixing, the matrices $T^{(m m)}(\beta)$ are irreducible and aperiodic for even $m$. The Perron-Frobenius Theorem (see, for example, [16, p.53] or [24, p.536]), now guarantees that $T^{(m m)}(\beta)$ has a simple, positive eigenvalue, larger (in modulus) than all other eigenvalues, which we denote by $\nu_{m}(\beta)$. Now, $\nu_{0}(\beta)$ determines the topological pressure, given by $P(\beta)=\ln \nu_{0}(\beta)$, which has the following well-known properties.

\section{Lemma 2.1.}

(i) $P(1)=0$;

(ii) $P(\beta)$ is a convex function of $\beta$;

(iii) $P(\beta)$ is analytic in $\beta$;

(iv) $P^{\prime}(1)=-\Lambda$.

These properties have been established for a rather large class of dynamical systems (see, for example, [20]). In our context, however, they follow easily from the matrix representation above using elementary methods. As an example, we shall derive property (ii). To simplify notation, we shall denote the first block matrix $T^{(00)}(\beta)$ by $T(\beta)$ for the remainder of the argument. Observing that $P(\beta)=$ $\ln \nu_{0}(\beta)$ is obtained from $\ln \operatorname{Tr}\left((T(\beta))^{n}\right) / n$ as $n$ tends to infinity, convexity of the topological pressure, that is,

$$
P\left(\beta_{1}(1-t)+\beta_{2} t\right) \leq(1-t) P\left(\beta_{1}\right)+t P\left(\beta_{2}\right) \quad(0 \leq t \leq 1),
$$

follows from a simple estimate using the Hölder inequality

$$
\begin{aligned}
& \frac{1}{n} \ln \operatorname{Tr}\left(\left(T\left(\beta_{1}(1-t)+\beta_{2} t\right)\right)^{n}\right) \\
= & \frac{1}{n} \ln \sum_{l_{0}, l_{1}, \ldots, l_{n-1}} T_{l_{0} l_{1}}\left(\beta_{1}(1-t)+\beta_{2} t\right) T_{l_{1} l_{2}}\left(\beta_{1}(1-t)+\beta_{2} t\right) \cdots T_{l_{n-1} l_{0}}\left(\beta_{1}(1-t)+\beta_{2} t\right) \\
= & \frac{1}{n} \ln \sum_{l_{0}, l_{1}, \ldots, l_{n-1}}\left(T_{l_{0} l_{1}}\left(\beta_{1}\right)\right)^{1-t}\left(T_{l_{0} l_{1}}\left(\beta_{2}\right)\right)^{t} \cdots\left(T_{l_{n-1} l_{0}}\left(\beta_{1}\right)\right)^{1-t}\left(T_{l_{n-1} l_{0}}\left(\beta_{2}\right)\right)^{t} \\
\leq & \frac{1}{n} \ln \left(\sum_{l_{0}, l_{1}, \ldots, l_{n-1}} T_{l_{0} l_{1}}\left(\beta_{1}\right) \cdots T_{l_{n-1} l_{0}}\left(\beta_{1}\right)\right)^{1-t}\left(\sum_{l_{0}, l_{1}, \ldots l_{n-1}} T_{l_{0} l_{1}}\left(\beta_{2}\right) \cdots T_{l_{n-1} l_{0}}\left(\beta_{2}\right)\right)^{t} \\
= & (1-t) \frac{1}{n} \ln \operatorname{Tr}\left(\left(T\left(\beta_{1}\right)\right)^{n}\right)+t \frac{1}{n} \ln \operatorname{Tr}\left(\left(T\left(\beta_{2}\right)\right)^{n}\right) .
\end{aligned}
$$

Here we have used that $(7)$ implies $T_{k l}\left(\beta_{1}+\beta_{2}\right)=T_{k l}\left(\beta_{1}\right) T_{k l}\left(\beta_{2}\right)$ as well as $T_{k l}(t \beta)=$ $\left(T_{k l}(\beta)\right)^{t}$. The other statements of the lemma can be derived in a similar way, or can be found in standard textbooks on ergodic theory.

To establish a relation between the correlation decay, that is, between the eigenvalues of the operator (5) for $\beta=1$, and the Lyapunov exponent, note that the largest eigenvalue of the Perron-Frobenius operator is given by $\nu_{0}(1)=1$, while $\nu_{2}(1)$ is a positive eigenvalue, which provides a lower bound for the subleading eigenvalue of $\mathcal{L}_{\beta}$. Thus, on the one hand

$$
\alpha_{\mathcal{H}} \leq-\ln \nu_{2}(1) .
$$

On the other hand $T^{(22)}(\beta)=T^{(00)}(\beta+2)$ by $(7)$, which implies

$$
\nu_{2}(\beta)=\nu_{0}(\beta+2) \text {. }
$$


Hence, using the properties of the topological pressure in Lemma 2.1, the relations (8) and (9) yield

$$
\alpha_{\mathcal{H}} \leq-P(3) \leq(3-1) \Lambda .
$$

See Figure 2 for a graphical illustration of this result.

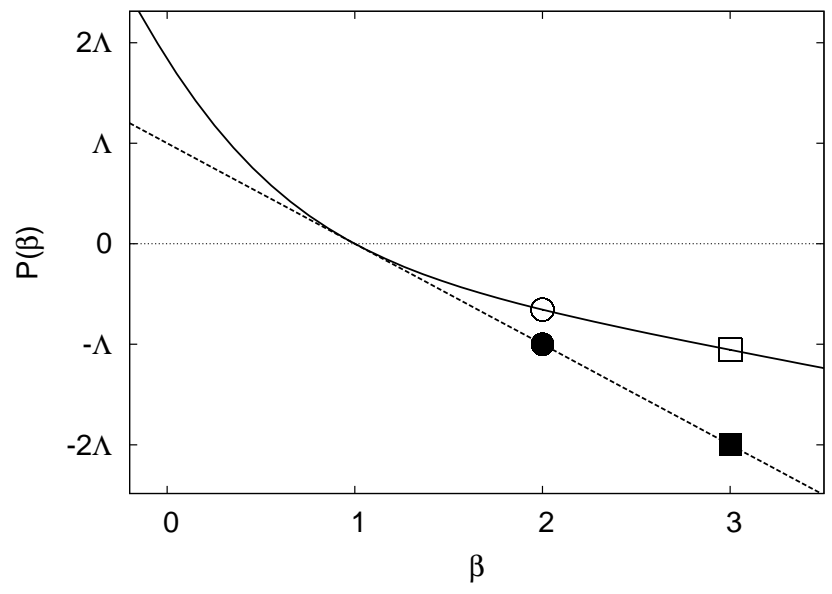

Figure 2. Schematic representation of the topological pressure $P(\beta)$ and graphical illustration of the estimates (10) and (11).

Note that if all slopes $\gamma_{k}$ of $f$ have the same sign, then we have $T^{(11)}(\beta)=$ $\operatorname{sign}\left(\gamma_{k}\right) T^{(00)}(\beta+1)$. Thus, we can apply the previous arguments to $\left|\nu_{1}(1)\right|$ to obtain the following improved estimate

$$
\alpha_{\mathcal{H}} \leq-\ln \left|\nu_{1}(1)\right|=-\ln \nu_{0}(2)=-P(2) \leq(2-1) \Lambda .
$$

To summarise, we have shown the following.

Proposition 2.2. Let $f: I \rightarrow I$ denote a topologically mixing piecewise linear expanding Markov map. If $\mathcal{H}=\mathcal{H}\left(D_{R}\right)$ denotes a space of piecewise analytic observables (see Appendix, Definition A.2) then the mixing rate is bounded in terms of the Lyapunov exponent $\Lambda$ with respect to the piecewise constant invariant density, by

$$
\alpha_{\mathcal{H}} \leq 2 \Lambda \text {. }
$$

If all slopes have the same sign the sharper estimate

$$
\alpha_{\mathcal{H}} \leq \Lambda
$$

holds.

Remark 2.3. The assumption that $f$ be topologically mixing is sufficient but not necessary. Indeed, there exist piecewise linear expanding Markov maps $f$ with the following properties: the map $f$ is not topologically mixing, yet exhibits exponential decay of correlations and the conclusions of Proposition 2.2 hold.

The simplest examples for which the bounds are achieved are the tent map $\left(\alpha_{\mathcal{H}}=2 \Lambda\right)$ and the doubling map $\left(\alpha_{\mathcal{H}}=\Lambda\right)$. It is finally worth mentioning that the condition $P(1)=0$, that is, the absolute continuity of the reference measure with respect to Lebesgue measure, is not essential for Proposition 2.2 to be valid. 
The conclusions also hold, for instance, for certain Gibbs measures with respect to piecewise constant potentials.

\section{REMARKS ON GENERAL EXPANDing Markov MAPS}

The setup of piecewise linear Markov maps is rather special. One may thus be tempted to ask whether a result like Proposition 2.2 extends, say, to Markov maps with finite curvature. The previous considerations are based on (9), a result which has been exploited previously in a slightly more restricted setup [2] to conjecture an exact relation between correlation decay and generalised Lyapunov exponents. It has already been demonstrated that such an identity breaks down for maps with finite curvature [11]. This fact, taken on its own, however, does not prevent the validity of a generalisation of Proposition 2.2, making a study of how curvature affects the previous considerations a worthy task.

Using an Ulam-like construction any expanding Markov map can be approximated by a piecewise linear map (see, for example, [4]). Let $F: I \rightarrow I$ denote a piecewise expanding, but not necessarily linear Markov map with Markov partition $\left\{I_{1}, \ldots, I_{N}\right\}$. Using the cylinder sets $U_{i_{0}, \ldots, i_{n-1}}=\cap_{k=0}^{n-1} F^{-k}\left(I_{i_{k}}\right)$ we may introduce a piecewise linear approximation $f_{n}: I \rightarrow I$ by the following construction. The map $f_{n}$ linearly interpolates $F$ on each cylinder set, that is, $f_{n}\left(U_{i_{0}, \ldots, i_{n-1}}\right)=U_{i_{1}, \ldots, i_{n-1}}$ (see Figure 3(a)). It is a straightforward exercise to show that the Lyapunov exponent of $f_{n}$ tends to the Lyapunov exponent of $F$ (with respect to the absolutely continuous invariant measure) as $n$ tends to infinity. However, the analysis of the mixing rate requires greater care. While Proposition 2.2 is still valid for any order $n$ of the approximation, it is far from obvious whether the proposition is valid for a general expanding map.

To illustrate this point we consider a simple example, a family of full branch piecewise Möbius maps $F_{c}$ defined on $[-1,1]$,

$$
F_{c}(x)=\frac{1-2(c+1)|x|}{1+2 c|x|} .
$$

We restrict the parameter to $c \in(-1 / 4,1 / 2)$, in order to guarantee expansivity. Figure $3(\mathrm{a})$ depicts the map $F_{c}$ for $c=-0.22$.

The leading part of the spectrum of the corresponding Perron-Frobenius operator considered on the space of analytic observables can be approximated using a spectral approximation method. The basic idea of this method is to approximate $\mathcal{L}_{\beta}$ by an $n \times n$ square matrix $\Pi_{n} \mathcal{L}_{\beta} \Pi_{n}$, where $\Pi_{n}$ denotes the projector that sends a function to its Lagrange-Chebyshev interpolating polynomial of degree $n-1$. This method is easily implemented and, moreover, it is possible to show (see [5]) that the eigenvalues of $\Pi_{n} \mathcal{L}_{\beta} \Pi_{n}$ converge exponentially fast to the eigenvalues of $\mathcal{L}_{\beta}$. Using this method the leading eigenvalues of the Perron-Frobenius operator and their dependence on $c$ are easily obtained (see Figure 3(b)). A minimum for the subleading eigenvalue occurs at about $c=-0.11$. The corresponding numerical value reads $\lambda_{1} \approx 0.10415$ resulting in a mixing rate $\alpha_{\mathcal{H}}=-\ln \left|\lambda_{1}\right| \approx 2.2619$. The corresponding Lyapunov exponent (which hardly depends on the parameter $c$ ) is computed using the numerical approximation of the invariant density. The numerical value is $\Lambda \approx 0.685$ so that the inequality (12) is clearly violated.

In order to understand why the reasoning at the beginning of this section fails, that is, why an approximation of the map $F_{c}$ by a piecewise linear Markov map 

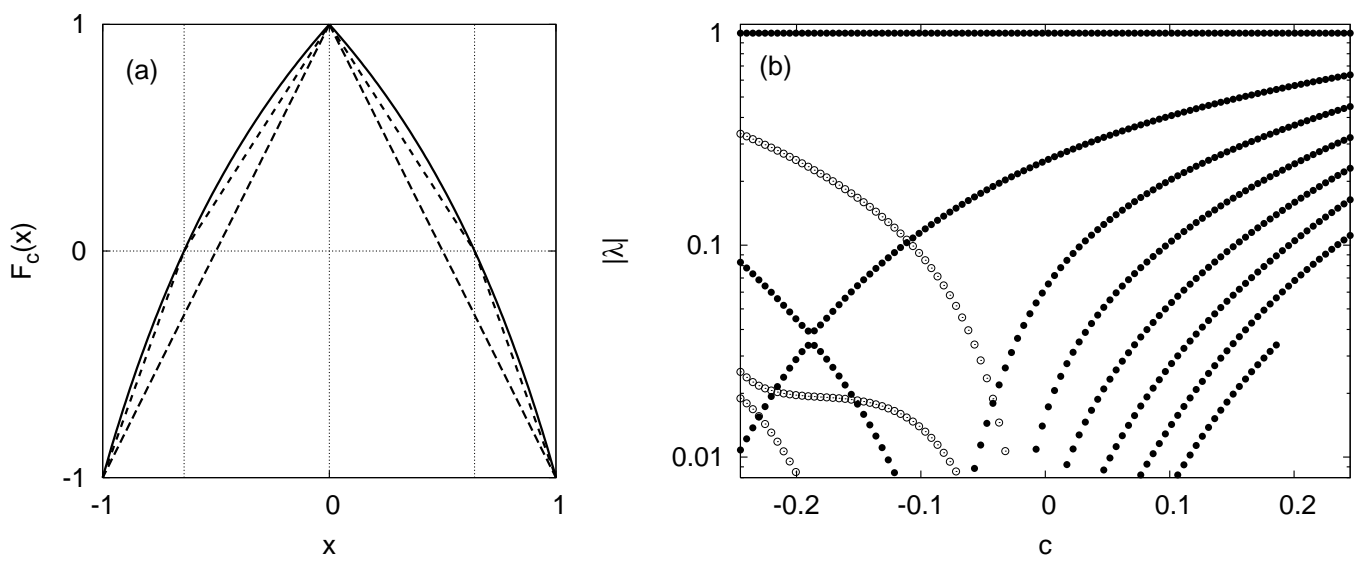

Figure 3. Left: (a) Diagrammatic view of the Möbius map (14) for $c<0$ (solid). Dotted lines indicate the cylinder sets of the first generations. Broken lines show the first two piecewise linear approximations $f_{1}$ and $f_{2}$.

Right: (b) The largest eigenvalues (in modulus) of the PerronFrobenius operator for the map $F_{c}$ in dependence on $c$, as obtained using the Lagrange-Chebyshev method with truncation order $n=25$. Positive/negative eigenvalues are indicated by filled/open symbols.

fails to produce the correct mixing rate, let us consider increasingly finer piecewise linear approximations $f_{n}$ of the map (14), see Figure 3(a). For each map $f_{n}$ the Perron-Frobenius operator restricted to piecewise polynomial functions has a finite matrix representation (see (6)). For the remainder of the section we shall only deal with the case $\beta=1$ and refer to $T(\beta)$ (and $\nu_{m}(\beta)$ ) as $T$ (and $\nu_{m}$ ). Figure 4(a) shows the numerical results for the leading eigenvalues of the diagonal block $T^{(11)}$ and $T^{(22)}$, that is, $\nu_{1}$ and $\nu_{2}$, respectively, for increasing level of approximation $n=1, \ldots, 6$. For comparison we display the subleading eigenvalue $\nu_{0}^{(s u b l)}$ of $T^{(00)}$ as well. For every level $n$ of approximation, $\nu_{2}$ gives the subleading eigenvalue $\lambda_{1}$ of the Perron-Frobenius operator of the piecewise linear approximation, and these values seem to converge as $n$ tends to infinity. The values and the limit are larger than $\exp (-2 \Lambda)$, meaning that the inequality (12) in the Proposition 2.2 is satisfied, as expected. The eigenfunction $u_{n}:[-1,1] \rightarrow \mathbb{R}$ of the corresponding eigenvalue $\nu_{2}$ is a quadratic polynomial on each element of the partition, but it develops an increasing number of discontinuities between different intervals of the increasingly finer partition. These eigenfunctions do not seem to converge to a smooth limit (see Figure 4(b)). In fact, unlike the invariant density there is no reason why the limit should be smooth. The numerical experiment suggests that we end up with a function of bounded variation.

It is indeed possible to show and perhaps well known that an estimate like (13) holds for discontinuous observables. For that purpose let us consider the PerronFrobenius operator $\mathcal{L}_{1}$ on the space of functions of bounded variation. Recall that a function $f:[-1,1] \rightarrow \mathbb{R}$ is of bounded variation if it has finite total variation 

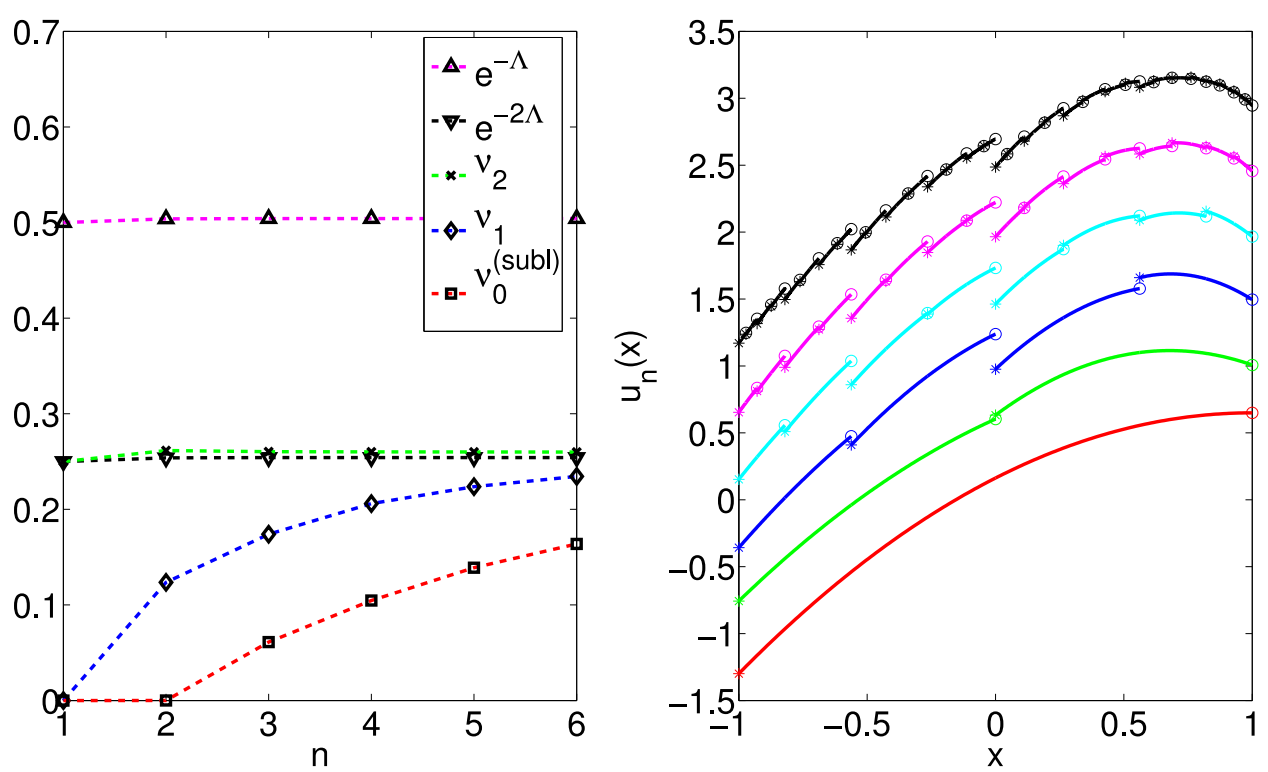

Figure 4. Left: (a) Leading eigenvalues $\nu_{1}$ and $\nu_{2}$ of the corresponding matrix blocks $T^{(11)}$ and $T^{(22)}$ for a piecewise linear approximation of the map (14) with $c=-0.11$, as a function of the level of approximation. The subleading eigenvalue of $T^{(00)}, \nu_{0}^{(s u b l)}$, is displayed as well. For comparison, $\exp (-\Lambda)$ and $\exp (-2 \Lambda)$ are depicted as well. The broken lines are a guide for the eye.

Right: (b) Eigenfunction $u_{n}$ corresponding to $\nu_{2}$ for $n=1, \ldots, 6$ with normalisation $\int_{I}\left|u_{n}(x)\right| d x=1$. For clarity, successive approximations are shifted by 0.5 . The open symbols indicate the discontinuity set of the eigenfunction, i.e., the increasingly finer Markov partition of the piecewise linear approximaton.

$\operatorname{var}(f)=\sup \left\{\sum_{i=1}^{p}\left|f\left(x_{i}\right)-f\left(x_{i-1}\right)\right|:-1 \leq x_{0} \leq \ldots \leq x_{p} \leq 1\right\}<\infty$. In this setup, the spectrum of the Perron-Frobenius operator associated with expanding maps has been studied in detail (see [19]). In particular, there is an explicit formula for the essential spectral radius given by

$$
\sigma_{\text {ess }}=\lim _{k \rightarrow \infty}\left(\inf \left\{\left|\left(F_{c}^{k}\right)^{\prime}(x)\right|: x \in[-1,1]\right\}\right)^{-1 / k} .
$$

Thus, we have an upper bound for the mixing rate

$$
\alpha_{B V} \leq-\ln \sigma_{e s s}=\lim _{k \rightarrow \infty} \frac{1}{k} \ln \inf \left\{\left|\left(F_{c}^{k}\right)^{\prime}(x)\right|: x \in[-1,1]\right\},
$$

which yields the following estimate for the Lyapunov exponent:

$$
\begin{aligned}
\Lambda & =\frac{1}{k} \int_{I} \ln \left|\left(F_{c}^{k}\right)^{\prime}(x)\right| d \mu \\
& \geq \frac{1}{k} \inf \left\{\ln \left|\left(F_{c}^{k}\right)^{\prime}(x)\right|: x \in[-1,1]\right\} \int_{I} d \mu \\
& =\frac{1}{k} \ln \inf \left\{\left|\left(F_{c}^{k}\right)^{\prime}(x)\right|: x \in[-1,1]\right\} .
\end{aligned}
$$


Thus, for observables of bounded variation we have the following result.

Proposition 3.1. Let $f: I \rightarrow I$ be a piecewise monotonic smooth expanding interval map which is mixing with respect to its unique absolutely continuous invariant measure. Then the rate of decay of correlations for functions of bounded variation is bounded by the Lyapunov exponent

$$
\alpha_{B V} \leq \Lambda .
$$

In fact, almost identical statements can be found in [12], for example, Corollary 9.2 .

\section{Conclusion}

There is no simple, straightforward answer to the question about the relation between Lyapunov exponents and mixing rates. On formal grounds one may argue that both quantities probe entirely different and independent aspects of a dynamical system, and that no particular relation should be expected. Lyapunov exponents are determined by properties related to the largest eigenvalue of the Perron-Frobenius operator. By contrast, correlation decay depends crucially on properties of the observables, with mixing rates being related to the subleading part of the spectrum. Thus, abstract operator theory on its own does not seem to provide further insight into the relation between both quantities. Witness, for example, the doubling map viewed as an analytic map on the unit circle. While its Lyapunov exponent is finite, the Perron-Frobenius operator has no nontrivial eigenvalue when considered on the space of analytic functions, that is, correlations of analytic observables decay faster than any exponential (see, e.g., [3]).

The argument outlined above, however, is a bit too simplistic. In fact, our results on piecewise linear expanding Markov maps observed via piecewise analytic functions or general piecewise smooth expanding maps observed via functions of bounded variation suggest that bounds on the mixing rate in terms of Lyapunov exponents can be derived provided that specific properties of the underlying dynamical system are taken into account. Estimates of this type rely on nontrivial lower bounds for spectra. As such, they are complementary to estimates which are available for proving the existence of spectral gaps, and will thus require completely different approaches.

It turns out that the spirit of the result contained in Proposition 3.1 can be understood by considering an observable with a single discontinuity. In order to substantiate this claim we have performed numerical simulations on the map (14) for $c=-0.11$. We have computed the autocorrelation function (4) for the observable $\varphi=\psi=\phi_{h}$ with $\phi_{h}(x)=x$ if $|x|<1 / 2$ and $\phi_{h}(x)=x-\operatorname{sign}(x) h$ if $|x|>1 / 2$, having a discontinuity of stepsize $h$ at $|x|=1 / 2$ (see Figure 5). Choosing $h=0$, the corresponding observable $\phi_{h}$ is analytic and the correlation decay is seen to follow the subleading eigenvalue $\lambda_{1}$ of the Perron-Frobenius operator defined on the space of analytic functions (see Figure $3(\mathrm{~b})$ ). In the discontinuous case corresponding to $h \neq 0$ the short time initial decay of the correlations still follows the pattern of the analytic observable, but the correlation function now develops an exponential tail which obeys (15). The tail becomes more pronounced if the stepsize increases. In fact, the mixing rate seems to be very close to the Lyapunov exponent. Revisiting the considerations leading to Proposition 3.1, it is tempting to surmise that this coincidence is a consequence of large deviation properties of finite time Lyapunov 
exponents, since the expression for the essential spectral radius involves an extreme value of a finite time Lyapunov exponent. Thus, the relation between Lyapunov exponents and mixing rates for observables of bounded variation could be viewed to arise from the same mechanism already exploited in [2] for analysing the simple case mentioned in the introduction.

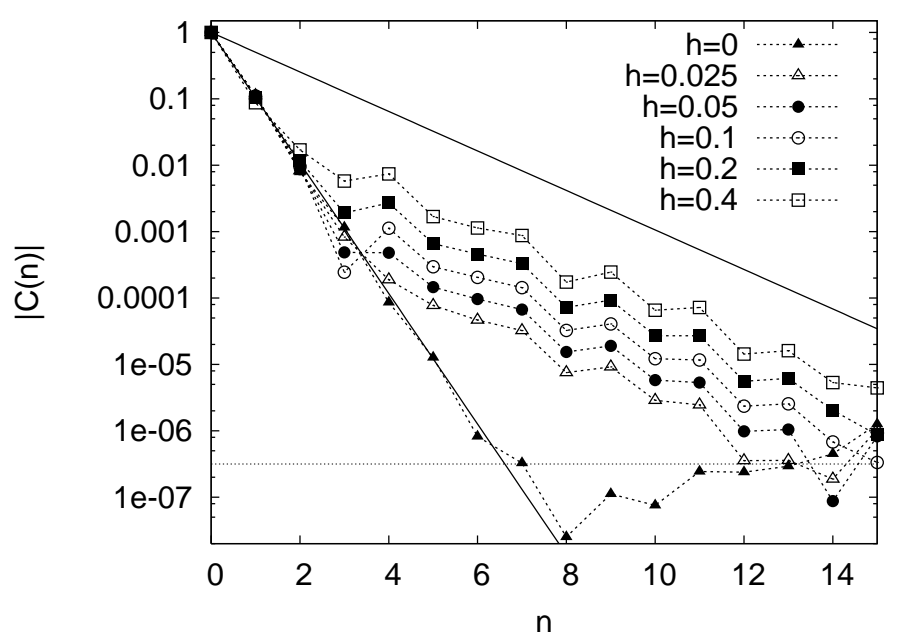

FiguRE 5. Normalised autocorrelation function of the discontinuous variable $\phi_{h}$ with different stepsizes $h$ for the map $F_{c}$ (14) with $c=-0.11$. The straight lines represent an exponential decay with rate $\ln \left|\lambda_{1}\right|$ and $-\Lambda$. Ergodic averages have been computed as time averages of a series of length $2 \times 10^{4}$ for $5 \times 10^{8}$ uniformly distributed initial conditions, skipping a transient of length 100 . The horizontal dotted line indicates the order of magnitude of statistical errors induced by the finite ensemble size.

This simple demonstration gives support to the folklore that correlation decay is linked with Lyapunov exponents. Even if a real world phenomenon is sufficiently well-modelled by a smooth dynamical system for which to date no link between correlation decay and Lyapunov exponents can be established, one should keep in mind that modern data processing inevitably involves digital devices, which correspond to discontinuous observations. Therefore, in formal terms observables of bounded variation could be the relevant class for applications and in these cases Proposition 3.1 applies.

At an intuitive level it is easy to understand why discontinuous observations result in correlation decay related to Lyapunov exponents. A discontinuous observable is able to distinguish between different "microstates" at a "macroscopic" level, that is, a discontinuous observation is able to distinguish two states at infinitesimal distance. As the distance between two nearby phase space points separated by a discontinuity grows according to the Lyapunov exponent, the sensitivity at the microscale may be transported to the macroscale, that is, it may filter through to the correlation function by a discontinuous observation. 
In our context the mathematical challenge is to establish a relation between mixing rates and Lyapunov exponents for natural classes of observables, for example, full branch analytic interval maps observed via analytic functions. Besides the need for developing tools to obtain lower bounds for spectra, establishing the relation alluded to above also requires a deeper understanding of which dynamical feature causes the point spectrum of the Perron-Frobenius operator, that is, which signature of the microscopic dynamics survives if viewed via analytic observables. This is reminiscent of coarse-graining approaches in statistical mechanics, for example, the introduction of collective coordinates and quasi-particles. Thus, tackling the mathematical problem above may well shed some light on some of the most fundamental problems in contemporary nonequilibrium statistical physics of complex systems.

\section{ACKNOWLEDGEMENTS}

W.J. gratefully acknowledges support by EPSRC (grant no. EP/H04812X/1) and DFG (through SFB910), as well as the kind hospitality by Eckehard Schöll and his group during the stay at TU-Berlin.

\section{APPENDIX: SPECTRAL PROPERTIES OF MARKOV MAPS}

In this section we shall provide a short account of the technical details to make the results of Section 2 rigorous. The main thrust of the argument is to define a suitable function space on which the generalised Perron-Frobenius operator is

compact. Results of this type for general analytic Markov maps are not new (see, for example, [29] or [26]). The special case of piecewise linear Markov maps discussed below, where a complete determination of the spectrum is possible, is probably well known to specialists in the field. Unfortunately, we are at loss to provide a reference for the results in Proposition A.5 below, so we will outline a proof for the convenience of the reader.

To set the scene we define what is meant by a piecewise linear Markov map. Before doing so we note that by a partition of a closed interval $I$ we mean a finite collection of closed intervals $\left\{I_{1}, \ldots, I_{N}\right\}$ with disjoint interiors, that is, $\operatorname{int}\left(I_{k}\right) \cap$ $\operatorname{int}\left(I_{l}\right)$ for $k \neq l$, such that $\bigcup_{k=1}^{N} I_{k}=I$.

Definition A.1. An interval map $f: I \rightarrow I$ is said to be a Markov map if there exists a finite partition $\left\{I_{k}\right\}_{k=1}^{N}$ of $I$ such that for any pair $(k, l)$ either $f\left(\operatorname{int}\left(I_{k}\right)\right) \cap$ $\operatorname{int}\left(I_{l}\right)=\emptyset$ or $\operatorname{int}\left(I_{l}\right) \subseteq f\left(\operatorname{int}\left(I_{k}\right)\right)$. If this is the case, the corresponding partition will be referred to as a Markov partition and the $N \times N$ matrix $A$ given by

$$
A_{k l}= \begin{cases}1 & \text { if } \operatorname{int}\left(I_{l}\right) \subseteq f\left(\operatorname{int}\left(I_{k}\right)\right) \\ 0 & \text { otherwise }\end{cases}
$$

will be called the topological transition matrix of the Markov map $f$.

A Markov map $f$ with Markov partition $\left\{I_{k}\right\}_{k=1}^{N}$ is said to be expanding if $\left|f^{\prime}(x)\right|>1$ for all $x \in \operatorname{int}\left(I_{k}\right)$. It is said to be piecewise linear if $f^{\prime}$ is constant on each element of the Markov partition, that is, $f^{\prime}(x)=\gamma_{k}$ for all $x \in \operatorname{int}\left(I_{k}\right)$. 
Finally, we call an expanding Markov map with topological transition matrix A topologically mixing ${ }^{1}$ if there is a positive integer $p$ such that each entry of the matrix $A^{p}$ is strictly positive.

In what follows we shall concentrate on topologically mixing piecewise linear expanding Markov maps. Our aim is to define suitable spaces of observables on which the associated generalised Perron-Frobenius operator or transfer operator

$$
\left(\mathcal{L}_{\beta} h\right)(x)=\sum_{y \in f^{-1}(x)} \frac{h(y)}{\left|f^{\prime}(y)\right|^{\beta}}
$$

(see (5)) is well defined and has nice spectral properties. It turns out that these spaces can be chosen from spaces of functions which are piecewise analytic.

In order to define these spaces we require some more notation.

Definition A.2. Let $D$ denote an open disk in the complex plane.

(i) We write $H^{\infty}(D)=\left\{h: D \rightarrow \mathbb{C}: h\right.$ holomorphic and $\left.\sup _{z \in D}|h(z)|<\infty\right\}$ to denote the space of bounded holomorphic functions on $D$. This is a Banach space when equipped with the norm $\|h\|_{H^{\infty}(D)}=\sup _{z \in D}|h(z)|$.

(ii) We use $\mathcal{H}(D)=\bigoplus_{k=1}^{N} H^{\infty}(D)$ to denote the space of $N$-tuples $\left(h_{1}, \ldots, h_{N}\right)$ of bounded holomorphic functions on $D$. This is a Banach space when equipped with the norm $\|h\|_{\mathcal{H}(D)}=\max \left\{\left\|h_{k}\right\|_{H^{\infty}(D)}: k=1, \ldots, N\right\}$.

The desired space of observables will now be defined by linking the disk $D$ occurring in the definition above to the dynamical system as follows. Given a piecewise linear expanding Markov map with Markov partition $\left\{I_{k}\right\}_{k=1}^{N}$, let us denote by $\varphi_{k l}: I_{k} \rightarrow I_{l}$ the inverse branch of the Markov map from partition element $I_{k}$ into the partition element $I_{l}$ as well as its obvious analytic continuation to the complex plane. Observe now that, since the map is expanding, all inverse branches are contractions. We can thus choose two concentric disks $D_{r}$ and $D_{R}$ of radius $r>0$ and $R>r$, respectively, such that

$$
\varphi_{k l}\left(D_{R}\right) \subset D_{r} \quad \text { for all inverse branches } \varphi_{k l} .
$$

It turns out that $\mathcal{H}\left(D_{R}\right)$ is a suitable space of observables for the map, in the sense that the associated transfer operator (17) is a well defined bounded operator on $\mathcal{H}\left(D_{R}\right)$. This is the content of the following result.

Proposition A.3. Given a piecewise linear expanding Markov map $f$ with topological transition matrix $A$ and inverse branches $\varphi_{k l}$, suppose that the disk $D_{R}$ is chosen as above. Then, for any real $\beta$, the transfer operator $\mathcal{L}_{\beta}$ is a well defined bounded operator from $\mathcal{H}\left(D_{R}\right)$ into itself and is given by

$$
\left(\mathcal{L}_{\beta} h\right)_{k}(z)=\sum_{l} A_{l k}\left|\varphi_{k l}^{\prime}(z)\right|^{\beta} h_{l}\left(\varphi_{k l}(z)\right) .
$$

Proof. The representation (19) follows from a short calculation using the definition (17) of $\mathcal{L}_{\beta}$. Since $\left|\varphi_{k l}^{\prime}(z)\right|^{\beta}=\left|\gamma_{l}\right|^{-\beta}$ is constant and the disk $D_{R}$ satisfies (18), the

\footnotetext{
${ }^{1}$ This is a slight abuse of terminology, since its use is usually restricted to continuous maps. However, it serves the same purpose as in the continuous setup as it guarantees the existence of a spectral gap for the corresponding transfer operator (see Corollary A.6).
} 
operator maps $\mathcal{H}\left(D_{R}\right)$ to $\mathcal{H}\left(D_{R}\right)$. In order to see that $\mathcal{L}_{\beta}: \mathcal{H}\left(D_{R}\right) \rightarrow \mathcal{H}\left(D_{R}\right)$ is bounded observe that if $h \in \mathcal{H}\left(D_{R}\right)$ with $\|h\|_{\mathcal{H}\left(D_{R}\right)} \leq 1$, then

$$
\left\|\mathcal{L}_{\beta} h\right\|_{\mathcal{H}\left(D_{R}\right)}=\max _{k}\left\|\left(\mathcal{L}_{\beta} h\right)_{k}\right\|_{H^{\infty}\left(D_{R}\right)} \leq \max _{k} \sum_{l} A_{l k}\left|\gamma_{l}\right|^{-\beta}<\infty .
$$

Remark A.4. The space $\mathcal{H}\left(D_{R}\right)$ is not the only suitable space of observables. Restricting to one and the same disk of analyticity for each branch, however, simplifies notation. More general spaces are discussed in [6] and [7].

Next we shall explain why the spectrum of $\mathcal{L}_{\beta}$ viewed as an operator on $\mathcal{H}\left(D_{R}\right)$ is given by the eigenvalues of the diagonal blocks $T^{(m m)}(\beta)$ for $m \in \mathbb{N}_{0}$ defined in (6).

The key ingredient of the proof of this statement is a factorisation of the transfer operator together with an approximation argument.

In order to explain the factorisation of the transfer operator we observe that in (19) the argument of $h_{l}$, that is $\varphi_{k l}(z)$, is contained in the smaller disk $D_{r}$ because of (18). We can thus use (19) to define the operator on a larger function space, namely $\mathcal{H}\left(D_{r}\right)$. Note that the space is 'larger' as analyticity is only required on a smaller disk $D_{r} \subset D_{R}$. We shall write $\tilde{\mathcal{L}}_{\beta}: \mathcal{H}\left(D_{r}\right) \rightarrow \mathcal{H}\left(D_{R}\right)$ in order to distinguish this lifted operator from the one occurring in Proposition A.3. Note that the arguments in this proposition can be adapted to show that $\tilde{\mathcal{L}}_{\beta}$ is a bounded operator.

It is tempting to think of $\mathcal{L}_{\beta}$ and $\tilde{\mathcal{L}}_{\beta}$ as being essentially the same, since they are given by the same functional expression. However, as operators the two are different as the latter is defined on a larger domain. Yet, both operators are related by restriction. In order to give a precise formulation of this fact we introduce a bounded embedding operator $\mathcal{J}$ which maps the smaller space $\mathcal{H}\left(D_{R}\right)$ injectively into the larger space $\mathcal{H}\left(D_{r}\right)$. To be precise $\mathcal{J}: \mathcal{H}\left(D_{R}\right) \rightarrow \mathcal{H}\left(D_{r}\right)$ is given by $(\mathcal{J} h)_{k}=J h_{k}$, where $J: H^{\infty}\left(D_{R}\right) \rightarrow H^{\infty}\left(D_{r}\right)$ in turn is given by $(J h)(z)=h(z)$ for $z \in D_{r}$. Note that $\mathcal{J}$ looks superficially like the identity. This, however, is misleading as argument and image are considered in different spaces.

The relation between $\mathcal{L}_{\beta}$ and $\tilde{\mathcal{L}}_{\beta}$ can now be written as

$$
\mathcal{L}_{\beta}=\tilde{\mathcal{L}}_{\beta} \mathcal{J} .
$$

Note that the factorisation above disentangles the intricacies of the map contained in $\tilde{\mathcal{L}}_{\beta}$ from its general expansiveness contained in $\mathcal{J}$.

We now turn to the approximation argument. For piecewise linear Markov maps the transfer operator is easily seen to map piecewise polynomial functions of degree at most $M$ into piecewise polynomial functions of degree at most $M$. This follows from a straightforward calculation using the fact that the inverse branches are affine functions.

In order to exploit this property of the transfer operator further we shall introduce a projection operator defined as follows: given an analytic function $h$ in $H^{\infty}\left(D_{R}\right)$ and an integer $M$ we use $P_{M} h$ to denote the truncated Taylor series expansion

$$
\left(P_{M} h\right)(z)=\sum_{k=0}^{M} \frac{h^{(k)}\left(z_{0}\right)}{k !}\left(z-z_{0}\right)^{k},
$$

where $z_{0}$ denotes the centre of the disk $D_{R}$. Clearly, $P_{M}$ is a projection operator. 
It turns out that the projections $P_{M}$ approximate the embedding $J$ for large $M$ in a strong sense. In order to make this statement, the heart of the approximation argument alluded to above, more precise, we observe that, by Cauchy's Integral Theorem, we have for any $h \in H^{\infty}\left(D_{R}\right)$ and any $z \in D_{r}$

$$
h(z)-\left(P_{M} h\right)(z)=\frac{1}{2 \pi i} \oint_{\Gamma} \frac{h(\zeta)}{\zeta-z} \frac{\left(z-z_{0}\right)^{M+1}}{\left(\zeta-z_{0}\right)^{M+1}} d \zeta,
$$

where the contour $\Gamma$ is the positively oriented boundary of a disk centred at $z_{0}$ with radius lying strictly between $r$ and $R$. It follows that the norm of $J-J P_{M}$ viewed as an operator from $H^{\infty}\left(D_{R}\right)$ to $H^{\infty}\left(D_{r}\right)$ satisfies

$$
\left\|J-J P_{M}\right\|_{H^{\infty}\left(D_{R}\right) \rightarrow H^{\infty}\left(D_{r}\right)} \leq \frac{R}{R-r}\left(\frac{r}{R}\right)^{M+1} .
$$

In particular, we have

$$
\lim _{M \rightarrow \infty}\left\|J-J P_{M}\right\|_{H^{\infty}\left(D_{R}\right) \rightarrow H^{\infty}\left(D_{r}\right)}=0 .
$$

In order to extend this result to the space of piecewise analytic functions $\mathcal{H}\left(D_{R}\right)$ we introduce the projection operator $\mathcal{P}_{M}: \mathcal{H}\left(D_{R}\right) \rightarrow \mathcal{H}\left(D_{R}\right)$ by setting $\mathcal{P}_{M} h=$ $\left(P_{M} h_{1}, \ldots, P_{M} h_{N}\right)$. The analogue of $(21)$ now reads

$$
\lim _{M \rightarrow \infty}\left\|\mathcal{J}-\mathcal{J} \mathcal{P}_{M}\right\|_{\mathcal{H}\left(D_{R}\right) \rightarrow \mathcal{H}\left(D_{r}\right)}=0 \text {. }
$$

We are now able to combine the factorisation (20) with the approximation result above to prove the main result of this Appendix.

Proposition A.5. Suppose we are given a piecewise linear expanding Markov map $f$ with inverse branches $\varphi_{k l}$ and disks $D_{r} \subset D_{R}$ satisfying (18). Then, for any real $\beta$, the transfer operator $\mathcal{L}_{\beta}$ viewed as an operator on $\mathcal{H}\left(D_{R}\right)$ is compact and its non-zero eigenvalues (with multiplicities) are precisely the non-zero eigenvalues of the matrices $T^{(m m)}(\beta)$ with $m \in \mathbb{N}_{0}$ given in (7).

Proof. We start by recalling that for every $M \geq 0$ the transfer operator $\mathcal{L}_{\beta}$ leaves the space $\mathcal{P}_{M}\left(\mathcal{H}\left(D_{R}\right)\right)$ invariant, that is, $\mathcal{L}_{\beta}\left(\mathcal{P}_{M}\left(\mathcal{H}\left(D_{R}\right)\right)\right) \subseteq \mathcal{P}_{M}\left(\mathcal{H}\left(D_{R}\right)\right)$. Thus

$$
\left(\mathcal{I}-\mathcal{P}_{M}\right) \mathcal{L}_{\beta} \mathcal{P}_{M}=0
$$

where $\mathcal{I}$ denotes the identity on $\mathcal{H}\left(D_{R}\right)$.

Using the above equation and the factorisation (20) we see that

$$
\begin{aligned}
& \left\|\mathcal{L}_{\beta}-\mathcal{P}_{M} \mathcal{L}_{\beta} \mathcal{P}_{M}\right\|_{\mathcal{H}\left(D_{R}\right)}=\left\|\mathcal{L}_{\beta}\left(\mathcal{I}-\mathcal{P}_{M}\right)\right\|_{\mathcal{H}\left(D_{R}\right)} \\
& \quad=\left\|\tilde{\mathcal{L}}_{\beta} \mathcal{J}\left(\mathcal{I}-\mathcal{P}_{M}\right)\right\|_{\mathcal{H}\left(D_{R}\right)} \leq\left\|\tilde{\mathcal{L}}_{\beta}\right\|_{\mathcal{H}\left(D_{r}\right) \rightarrow \mathcal{H}\left(D_{R}\right)}\left\|\mathcal{J}-\mathcal{J} \mathcal{P}_{M}\right\|_{\mathcal{H}\left(D_{R}\right) \rightarrow \mathcal{H}\left(D_{r}\right)},
\end{aligned}
$$

which, using (22), implies

$$
\lim _{M \rightarrow \infty}\left\|\mathcal{L}_{\beta}-\mathcal{P}_{M} \mathcal{L}_{\beta} \mathcal{P}_{M}\right\|_{\mathcal{H}\left(D_{R}\right)}=0 .
$$

Since $\mathcal{P}_{M} \mathcal{L}_{\beta} \mathcal{P}_{M}$ is a finite-rank operator for every $M$, the limit above implies that $\mathcal{L}_{\beta}$ is compact. Clearly, the non-zero eigenvalues of each $\mathcal{P}_{M} \mathcal{L}_{\beta} \mathcal{P}_{M}$ are exactly the non-zero eigenvalues of the block matrices (6). The remaining assertion, namely that the non-zero spectrum of the transfer operator is captured by the non-zero spectra of the finite dimensional matrix representations follows from (23) together with an abstract spectral approximation result (see [14, XI.9.5]).

Specialising to topologically mixing Markov maps we obtain the following refinement of the above proposition. 
Corollary A.6. Suppose that the hypotheses of the previous proposition hold. If the Markov map $f$ is also topologically mixing, then $\mathcal{L}_{\beta}: \mathcal{H}\left(D_{R}\right) \rightarrow \mathcal{H}\left(D_{R}\right)$ has a simple positive leading eigenvalue $\nu_{0}(\beta)$. Moreover, this leading eigenvalue is the Perron eigenvalue of the matrix $T^{(00)}(\beta)$.

Proof. This follows from the previous proposition together with the observation that for $m \geq 1$ the spectral radius $r\left(T^{(m m)}(\beta)\right)$ of the matrix $T^{(m m)}(\beta)$ is strictly smaller than the Perron eigenvalue of $T^{(00)}(\beta)$. In order to see this note that for all $m \geq 1$ we have

$$
\left|T_{k l}^{(m m)}(\beta)\right| \leq C T_{k l}^{(00)}(\beta),
$$

where

$$
C=\frac{1}{\inf _{l}\left|\gamma_{l}\right|}<1
$$

A short calculation shows that for each $k \geq 0$ and each $m \geq 1$ we have

$$
\left\|\left(T^{(m m)}(\beta)\right)^{k}\right\|_{F} \leq C^{k}\left\|\left(T^{(00)}(\beta)\right)^{k}\right\|_{F}
$$

where $\|\cdot\|_{F}$ denotes the Frobenius norm. The spectral radius formula now implies that

$$
r\left(T^{(m m)}(\beta)\right) \leq C \nu_{0}(\beta) .
$$

\section{REFERENCES}

[1] H. D. Abarbanel. Analysis of Observed Chaotic Data (Institute for Nonlinear Science). Springer, 1997.

[2] R. Badii, K. Heinzelmann, P. Meier, and A. Politi. Correlation functions and generalized Lyapunov exponents. Physical Review A, 37(4):1323-1328, Feb. 1988.

[3] V. Baladi. Positive transfer operators and decay of correlations. World Scientific Publishing Co. Pte. Ltd, Singapore, 2000.

[4] V. Baladi, S. Isola, and B. Schmitt. Transfer operator for piecewise affine approximations of interval maps. Annales de l'Institut Henri Poincaré (A) Physique théorique, 62(3):251-265, 1995.

[5] O. F. Bandtlow. Lagrange-Chebyshev approximation of transfer operators. In preparation.

[6] O. F. Bandtlow and O. Jenkinson. Explicit eigenvalue estimates for transfer operators acting on spaces of holomorphic functions. Advances in Mathematics, 218(3):902-925, June 2008.

[7] O. F. Bandtlow and O. Jenkinson. On the Ruelle eigenvalue sequence. Ergodic Theory and Dynamical Systems, 28(06):1701-1711, Dec. 2008.

[8] T. Bohr and D. Rand. The entropy function for characteristic exponents. Physica D: Nonlinear Phenomena, 25(1-3):387-398, Mar. 1987.

[9] A. Boyarsky and P. Gora. Laws of Chaos: Invariant Measures and Dynamical Systems in One Dimension (Probability and its Applications). Birkhäuser, 1997.

[10] H. Bruin, S. Luzzatto, and S. van Strien. Decay of correlations in one-dimensional dynamics. Annales Scientifiques de l'École Normale Supérieure, 36(4):621-646, July 2003.

[11] F. Christiansen, G. Paladin, and H. H. Rugh. Determination of correlation spectra in chaotic systems. Physical Review Letters, 65(17):2087-2090, Oct. 1990.

[12] P. Collet and J. P. Eckmann. Liapunov Multipliers and Decay of Correlations in Dynamical Systems. Journal of Statistical Physics, 115(1/2):217-254, Apr. 2004.

[13] J. D. Crawford and J. R. Cary. Decay of correlations in a chaotic measure-preserving transformation. Physica D: Nonlinear Phenomena, 6(2):223-232, Jan. 1983.

[14] N. Dunford and J. T. Schwartz. Linear Operators, Part 2: Spectral Theory. WileyInterscience, 1963.

[15] M. Falcioni, S. Isola, and A. Vulpiani. Correlation functions and relaxation properties in chaotic dynamics and statistical mechanics. Physics Letters A, 144(6-7):341-346, Mar. 1990.

[16] F. R. Gantmacher. Matrix Theory, Vol. 2. American Mathematical Society, 2000. 
[17] W. Just. Analytical treatment of fluctuation spectra at the symmetry breaking chaos transition. Physics Letters A, 150(8-9):362-368, Nov. 1990.

[18] H. Kantz and T. Schreiber. Nonlinear Time Series Analysis. Cambridge University Press, 2004.

[19] G. Keller. On the rate of convergence to equilibrium in one-dimensional systems. Communications in Mathematical Physics, 96(2):181-193, 1984.

[20] G. Keller. Equilibrium States in Ergodic Theory. Cambridge University Press, 1998.

[21] G. Keller. Interval maps with strictly contracting Perron-Frobenius operators. International Journal of Bifurcation and Chaos [in Applied Sciences and Engineering], 09:1777-1783, Sept. 1999.

[22] N. S. Krylov. Works on the Foundations of Statistical Physics (Princeton Series in Physics). Princeton University Press, 1979.

[23] R. Kubo. Brownian motion and nonequilibrium statistical mechanics. Science (New York, N.Y.), 233(4761):330-334, July 1986.

[24] P. Lancaster. Theory of Matrices. Academic Press Inc, 1969.

[25] C. Liverani. Decay of Correlations. The Annals of Mathematics Second Series, 142(2):239301, 1995.

[26] D. H. Mayer. Approach to equilibrium for locally expanding maps in $\mathbb{R}^{k}$. Communications in Mathematical Physics, 95(1):1-15, 1984.

[27] H. Mori, B.-C. So, and T. Ose. Time-Correlation Functions of One-Dimensional Transformations. Progress of Theoretical Physics, 66(4):1266-1283, Oct. 1981.

[28] F. Naud. Entropy and Decay of Correlations for Real Analytic Semi-Flows. Annales Henri Poincaré, 10(3):429-451, May 2009.

[29] D. Ruelle. Zeta-functions for expanding maps and Anosov flows. Inventiones Mathematicae, 34(3):231-242, Oct. 1976.

[30] B. C. So, N. Yoshitake, H. Okamoto, and H. Mori. Correlations and spectra of an intermittent chaos near its onset point. Journal of Statistical Physics, 36(3-4):367-400, Aug. 1984.

[31] J. C. Sprott. Chaos and Time-Series Analysis. Oxford University Press, 2001.

School of Mathematical Sciences, Queen Mary University of London, Mile End Road, LONDON E1 4NS, UK

E-mail address: j.slipantschuk@qmul.ac.uk, o.bandtlow@qmul.ac.uk, w.just@qmul.ac.uk 